Communications in Physics, Vol. 28, No. 4 (2018), pp. 301-310

DOI:10.15625/0868-3166/28/4/13195

\title{
SPONTANEOUS SYMMETRY BREAKING OF SOLITONS TRAPPED IN A DOUBLE-GAUSS POTENTIAL
}

\author{
NGUYEN DUY CUONG ${ }^{1,2, \dagger}$, DINH XUAN KHOA ${ }^{1}$, CAO LONG VAN ${ }^{3}$, M. \\ TRIPPENBACH ${ }^{4,5}$, BUI DINH THUAN ${ }^{1}$, AND DO THANH THUY ${ }^{1}$ \\ ${ }^{1}$ Vinh University, 182 Le Duan Street, Vinh City, Vietnam \\ ${ }^{2}$ Industrial University of Vinh, 26 Nguyen Thai Hoc Street, Vinh City, Vietnam \\ ${ }^{3}$ University of Zielona Góra, ul. Licealna 9, 65-417 Zielona Góra, Poland \\ ${ }^{4}$ Institute of Theoretical Physics, Physics Department, Warsaw University, Hoza 69, PL-00-681 \\ Warsaw, Poland \\ ${ }^{5}$ Soltan Institute for Nuclear Studies, Hoża 69, PL-00-681 Warsaw, Poland \\ ${ }^{\dagger}$ E-mail: duycuonghui@gmail.com
}

Received 16 October 2018

Accepted for publication 29 November 2018

Published 15 December 2018

\begin{abstract}
We consider an extended model of the previously considered model with double-square potential which is namely one-dimensional (1D) nonlinear Schrödinger equation (NLSE) with self-focusing nonlinearity and a $1 D$ double-gauss potential. Spontaneous symmetry breaking has been presented in terms of the control parameter which is propagation constant in the case of optics and chemical potential in the case of Bose-Einstein Condensate (BEC), correspondingly. The numerical simulations predict a bifurcation breaking the symmetry of $1 D$ solitons trapped in the double-gauss potential of the supercritical type as in the case of double-square potential. Furthermore we have constructed bifurcation diagrams, considering the stability of solitons with three methods including the method using Vakhitov-Kolokolov (V-K) Stability Criterion, Pseudospectral Method and Method for Linear-Stability Eigenvalues. It will show that the obtained results of our model from the three methods are the same but the third one is the fastest.
\end{abstract}

Keywords: Bose-Einstein condensate, nonlinear optical systems, double-square potential.

Classification numbers: 42.82.Et.

(C)2018 Vietnam Academy of Science and Technology 


\section{INTRODUCTION}

It is well-known that in the traditional quantum mechanics with Schrödinger equation as a fundamental one, the ground state of the linear system follows exactly the symmetry of the Hamiltonian describing the system. In contrast to this, the nonlinear terms in so-called nonlinear Schrödinger equation (NLSE) which describes the light propagation in nonlinear optical media and also BEC (named frequently as Gross-Pitaevskii Equation (GPE)) lead to spontaneous symmetry breaking (SSB), when ground states have other symmetry than Hamiltonian (trap potential) has. This phenomenon has been emphasized at the first time in 1979 by E. B. Davies [1] for atomic, molecular systems and analyzed intensively by B. A. Malomed's group since more than two decades [2-14]. In optics, the SSB occurs as a result of the interplay between the nonlinearity and waveguiding structures, when the strong nonlinearity partly suppresses the linear coupling between parallel guiding cores, for example in self-focusing Kerr medium [15]. The onset of a sharp symmetry-breaking instability in a double-hump two-component spatial optical

soliton was demonstrated experimentally in a planar nonlinear waveguide [16]. The analysis of the SSB for soliton modes was performed in models of dual-core fiber Bragg gratings with the Kerr nonlinearity [17], and coupled waveguides with the quadratic [18] and cubic-quintic [19] nonlinear terms. As it has been emphasized above, in the quantum world, a similar situation occurs when the double-well potential (DWP) traps an ultracold rarefied atomic gas in the state of the Bose-Einstein condensate (BEC). In the mean-field approximation, which is extremely accurate for rarefied atomic gases, repulsive or attractive collisions between atoms give rise to a cubic nonlinearity that emulates the optical Kerr effect. The outcome in this scenario is that the GrossPitaevskii equation (GPE) replaces the linear Schrödinger equation for describing the system [20]. In the effectively one-dimensional geometry, the SSB can be studied in the framework of the scaled NLSE/GPE with potential $V(x)$ of the DWP type, for the amplitude of the electromagnetic wave, or the single-particle wave function, $\psi(x, t)$ :

$$
i \frac{\partial \psi}{\partial t}=-\frac{1}{2} \psi_{x x}+\sigma|\psi|^{2} \psi+V(x) \psi
$$

where $t$ is the time in the GPE or the propagation distance in the NLSE $\sigma$ is nonlinear parameter, $\sigma=+1$ and $\sigma=-1$ are the self-repulsive and self-attractive cases in BEC (or the self-defocusing and self-focusing cases in optics), respectively. The $\psi_{x x}$ is second derivative of $\psi(x, t)$ with respect to $x$.

The analysis of the SSB in BEC and similar models based on Eq. (1) was initiated in Refs. [21] and [22]. Further, GPE (1) was extended by adding an extra (free) spatial coordinate, which transforms the DWP into a two-dimensional dual-core structure [23]. In such a setting, the self-attractive nonlinearity gives rise to matter-wave solitons, which are self-trapped in the free direction [24]. The SSB destabilizes symmetric solitons and replaces them by asymmetric ones, provided that the norm of the wave function (which determines the effective strength of the intrinsic nonlinearity) exceeds a critical value [23]. In the latter case, the mean-field symmetry breaking is a phase transition of the first kind (alias a subcritical bifurcation [22]), which includes hysteresis. The subcritical transition is typical to solitons in dual-core waveguides with the Kerr self-focusing [25-28]. The same type of the transition may be featured by CW (continuous-wave) states in dual-core systems with non-Kerr nonlinearities [29]. 
The present paper addresses the symmetry-breaking bifurcation and the existence and stability of symmetric states in a one-dimensional system, which is a direct extension of the familiar double-well model [23]. In this work, we focus on such effects in double-gauss potential settings, which can be engineered by means of techniques mentioned above, using the self-focusing nonlinearity in optics or attractive interactions between atoms in BEC, as briefly described below. In Sec. II, we formulate the model and give estimates of characteristic values of related physical parameters. Section III reports full numerical results of 1D model, that is the diagram of them and their stability is studied by means of direct simulations of slightly perturbed stationary states and also, independently, through the computation of respective stability eigenvalues for small perturbations. At the same time, we analyze the 1D model for double-rectangular potential considered in [23]. Section IV contains conclusions.

\section{THE MODEL}

Following Ref. [30], the generally scaled form of the 1D equation with the stripe-shaped potential and nonlinear parameter $\sigma=-1$ can be written as:

$$
i \frac{\partial \psi}{\partial t}=-\frac{1}{2} \psi_{x x}-|\psi|^{2} \psi+V(x) \psi
$$

In this work, we consider the case of double-gauss potential:

$$
V(x)=-\frac{1}{a \sqrt{\pi}}\left[\exp \left(-\frac{(x+1)^{2}}{a^{2}}\right)+\exp \left(-\frac{(x-1)^{2}}{a^{2}}\right)\right] .
$$

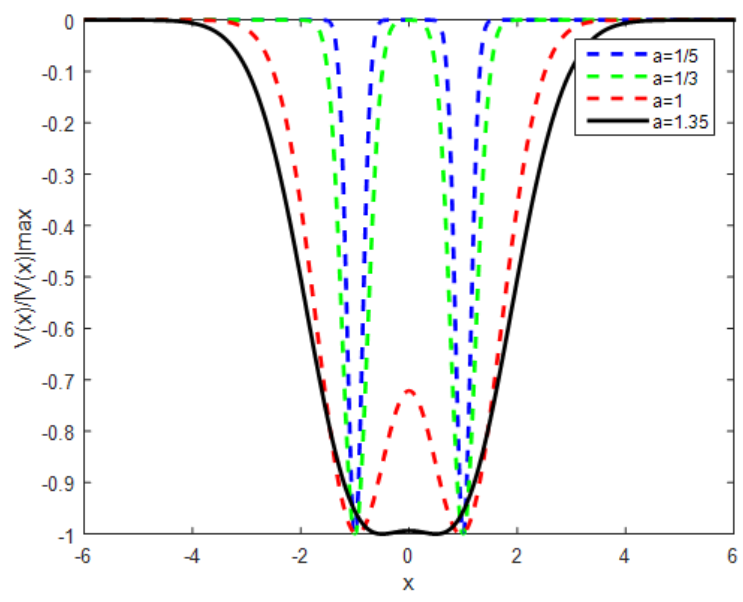

(a)

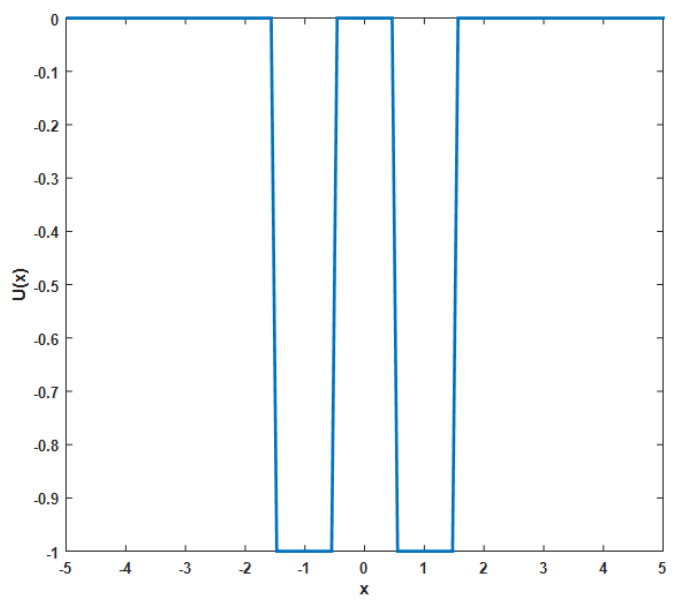

(b)

Fig. 1. The shape of one-dimensional potential $V(x),(a)$ corresponds to double-gauss potential for different values of scaled width a of the individual well; (b) The shape of the one-dimensional double-well potential $U(x)$ with $D, U_{0}$, and $L$ being, respectively, the width, depth of each well, and the width of the barrier between them (we will recall in title III.1). 
We are looking for stationary solutions as $\psi(x, t)=u(x) e^{-i \mu t}$ where $\mu$ is propagation constant (in optics) or chemical potential (in BEC) and real function $u(x)$ satisfies the equation:

$$
\mu u+\frac{1}{2} u_{x x}-V(x) u+u^{3}=0
$$

Equation (4) conserves a dynamical invariant, namely the norm:

$$
\begin{aligned}
N & =\int_{-\infty}^{+\infty}|\psi(x, t)|^{2} d x \\
& =\int_{-\infty}^{+\infty}|u(x)|^{2} d x .
\end{aligned}
$$

As in the previous papers [Refs. 4, 6, 11, 14], the asymmetry of soliton is defined by:

$$
\begin{aligned}
\Theta & =\frac{N_{+}-N_{-}}{N} \\
& =\frac{\left(\int_{0}^{+\infty}|u(x)|^{2} d x-\int_{-\infty}^{0}|u(x)|^{2} d x\right)}{\int_{-\infty}^{+\infty}|u(x)|^{2} d x} .
\end{aligned}
$$

\section{NUMERICAL RESULTS FOR BIFURCATION DIAGRAMS AND STABILITY}

\section{III.1. The one-dimensional setting for rectangular-well potentials}

We solved equation (4) numerically by using a new numerical code based on the Accelerated Imaginary-Time Evolution Method (AITM) with a fourth-order Runge-Kutta algorithm [30]. The accuracy of the numerical code was tested by comparing these results with the results obtained in the previous paper [23]. It follows that one can reconstruct all the results obtained in that paper.

In addition, in the case of double-rectangle potential $U(x)$ (see Fig. 1b) we also consider the stability of symmetry and asymmetry states. The instability of states is determined by two methods (direct propagation of perturbed states in real time method and $(V-K)$ Stability Criterion method [30]). The results are exactly the same. Note that, according to $(V-K)$ Criterion, if $N^{\prime}<0$ then the state is stable and if $N^{\prime}>0$ then the state is unstable (where the quantity $N^{\prime}$ is derivative of $N$ with respect to $\mu$ ).

In Fig. 2, the stability of solitons obtained by this method was then tested by direct propagation of perturbed states in real time. Perturbations which are actually large, were not able to destroy solitons that were identified as stable ones. On the contrary, much smaller perturbations were sufficient to demonstrate instability of those solutions which are unstable, after a propagation time of $t=1000$.

Now we present the results for the case of double-gauss well potential. Based on the code verified above, we set up bifurcation diagram of the symmetry breaking in this model. The results are illustrated in Fig. 3 below.

We set up bifurcation diagram for different width values. Figures 3(a), 3(b) and 3(c) corresponds with $a=0.2$, in this case, the bifurcation point is $N_{b i f}=0.65$; Figures 3(d), 3(e) and 3(f) corresponds with $a=0.5$, in this case, the bifurcation point is $N_{b i f}=0.925$; Figures $3(\mathrm{~g}), 3(\mathrm{~h})$ and 3(i) corresponds with $a=1$, in this case, the bifurcation point is $N_{b i f}=1.925$. Thanks to the illustration, we realize that the spontaneous symmetry breaking bifurcation is supercritical. We 


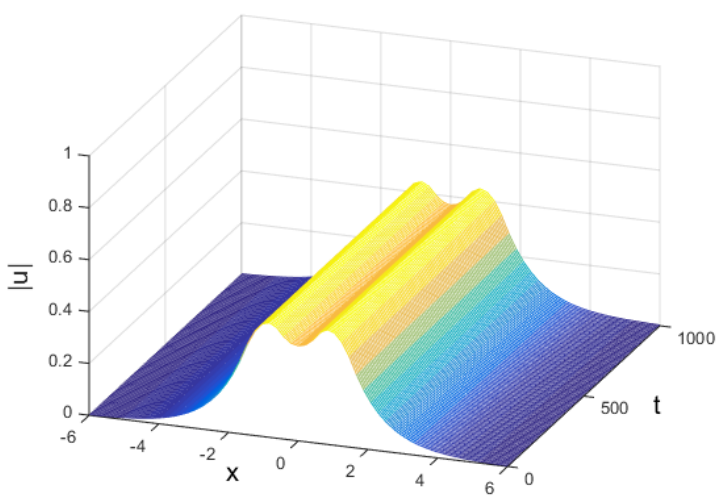

(a)

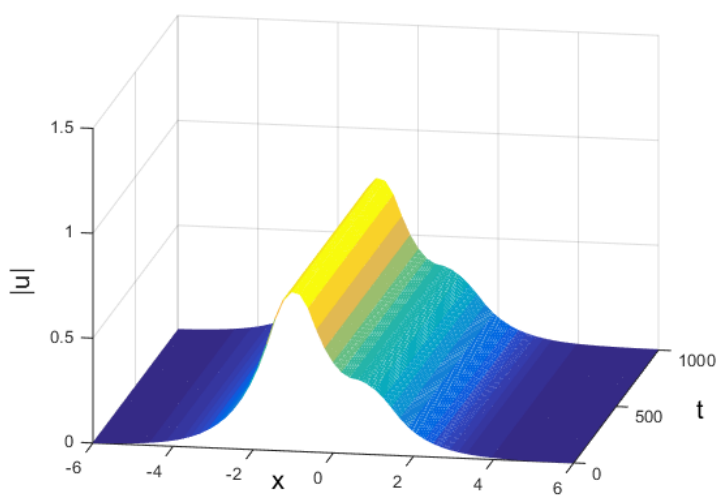

(b)

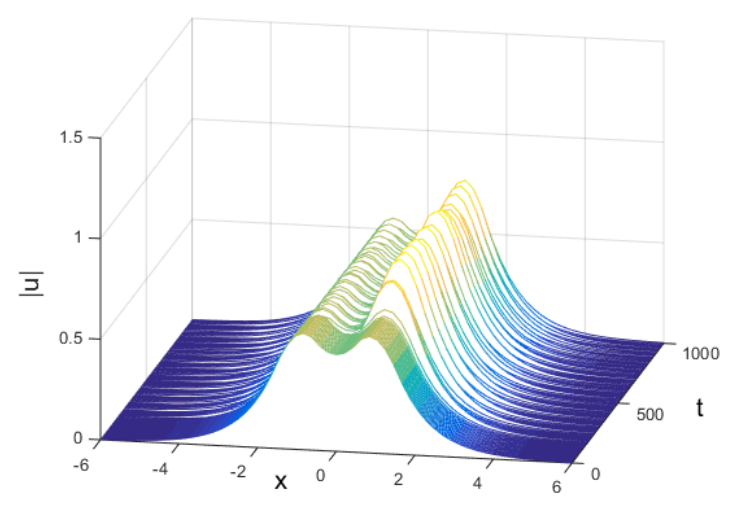

(c)

Fig. 2. The evolution of a stable symmetric state for $N=0.5$ (a), of a stable asymmetric state for $N=1$ (b), of an unstable symmetric state for $N=1$ (c). The parameters are $L=1, D=1, U=1$. The evolution time is $t=1000$.

can conclude that the results for smooth Gaussian potential agree with that for the rectangularwell model, what is according with our predictions. In the above figures, solid and dashed lines correspond to stable and unstable states.

In order to determine instability of soliton, we use Pseudospectral Method, (V-K) Criterion or use Methods for Linear-Stability Eigenvalues, all of them are described in [31].

In figures 3(c), 3(f), 3(i) we see that the slope of these curves is negative (it means $N^{\prime \prime}$ ). Thus these asymmetric states is stable.

Through the evolution of states from time to time, we will determine their stability. Figures. 4(a), 4(b), and 4(c) show the evolution of a symmetric state for $N=0.5, a=0.5$, the asymmetric state for $N=2, a=0.5$ and a symmetric state for $N=2, a=0.5$, respectively. Looking at the above figures, we lead to that two-peak symmetry soliton in Fig. 4(a), single-peak asymmetry soliton in Fig. 4(b) are stable and two-peak symmetry soliton in Fig. 4(c) is unstable. 


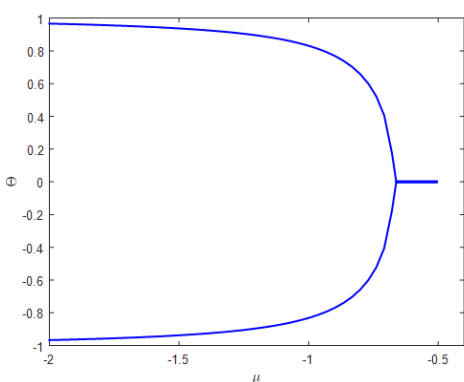

(a)

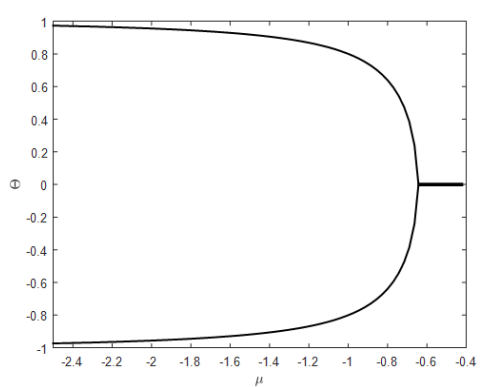

(d)

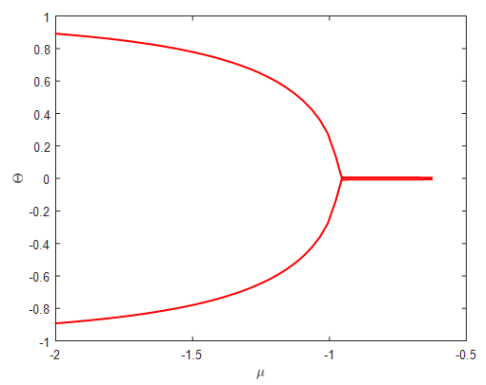

$(\mathrm{g})$

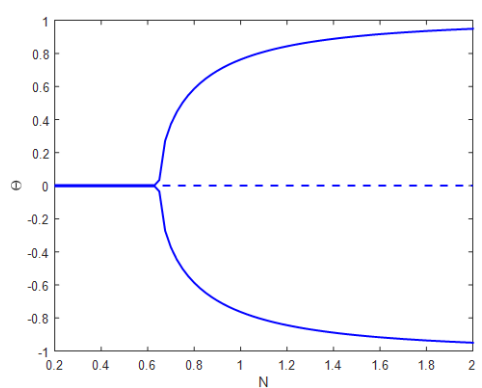

(b)

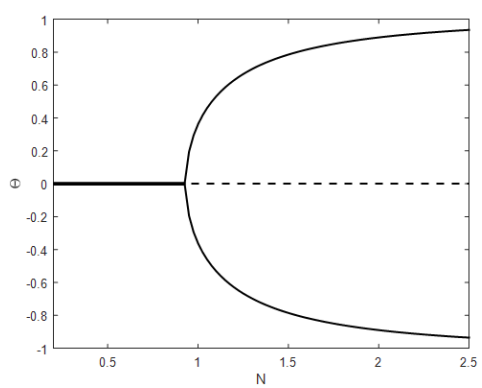

(e)

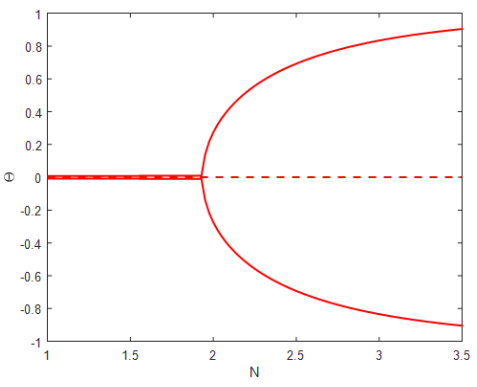

(h)

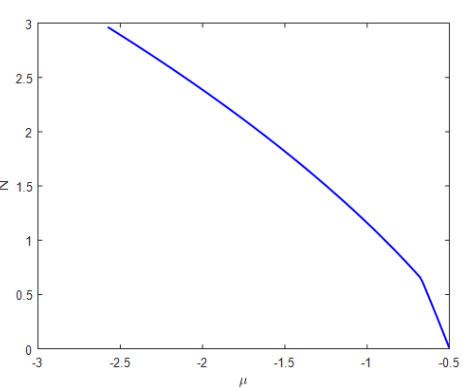

(c)

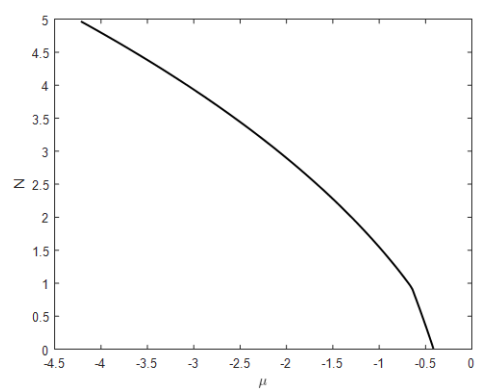

(f)

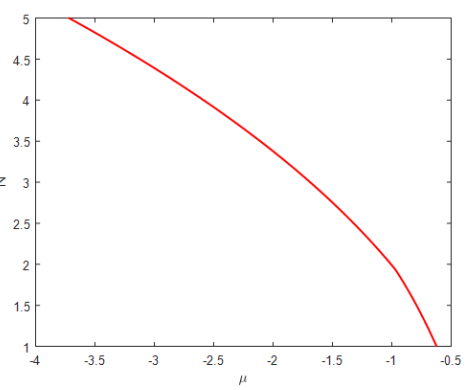

(i)

Fig. 3. (Color online) Families of asymmetric states in the plane of $(\Theta, \mu),(\Theta, N)$ and $(N, \mu):$ (a), (b), (c) for the values of potential parameter $a=0.2$; (d), (e), (f) for the values of potential parameter $a=0.5 ;(\mathrm{g})$, (h), (i) the values of potential parameter $a=1$.

For the computation of eigenvalues, perturbed solutions were looked for as

$$
\psi(x, t)=\left\{u(x)+[\mathscr{V}(x)+\mathscr{W}(x)] e^{\lambda t}+\left[\mathscr{V}^{*}(x)+\mathscr{W}^{*}(x)\right] e^{\lambda^{*} t}\right\} e^{-i \mu t}
$$

where $u(x)$ is a stationary solution to Eq. (4) with chemical potential (or is propagation constant) $\mu$, while $\mathscr{V}$ and $\mathscr{W}$ are components of a perturbation mode, pertaining to instability growth rate $\lambda \equiv \lambda_{r}+i \lambda_{i}$. The substitution of expression (7) into Eq. (2) and linearization lead to the eigenvalue 
problem based on the following equations:

$$
i\left(\begin{array}{cc}
0 & \frac{1}{2} \frac{d^{2}}{d x^{2}}+\mu+u^{2}(x)-V(x) \\
\frac{1}{2} \frac{d^{2}}{d x^{2}}+\mu+3 u^{2}(x)-V(x) & 0
\end{array}\right)\left(\begin{array}{c}
V \\
\mathscr{W}
\end{array}\right)=\lambda\left(\begin{array}{l}
\mathscr{V} \\
\mathscr{W}
\end{array}\right)
$$

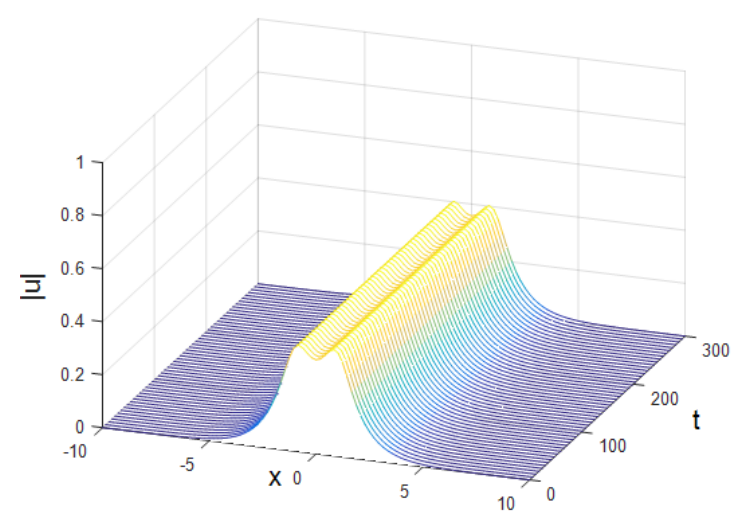

(a)

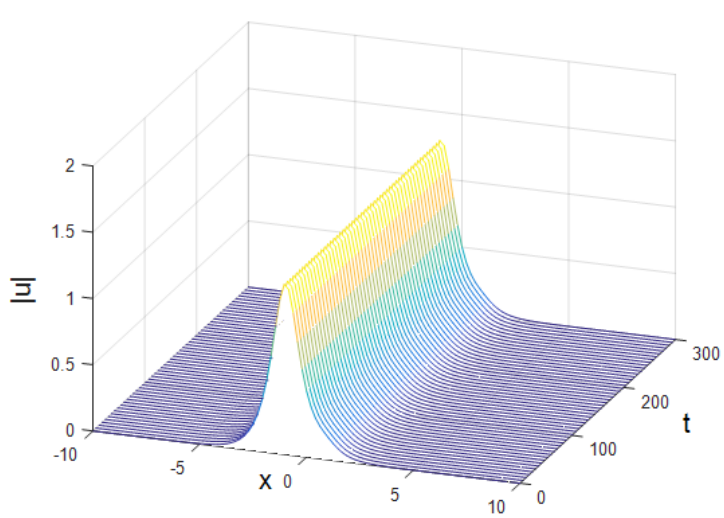

(b)

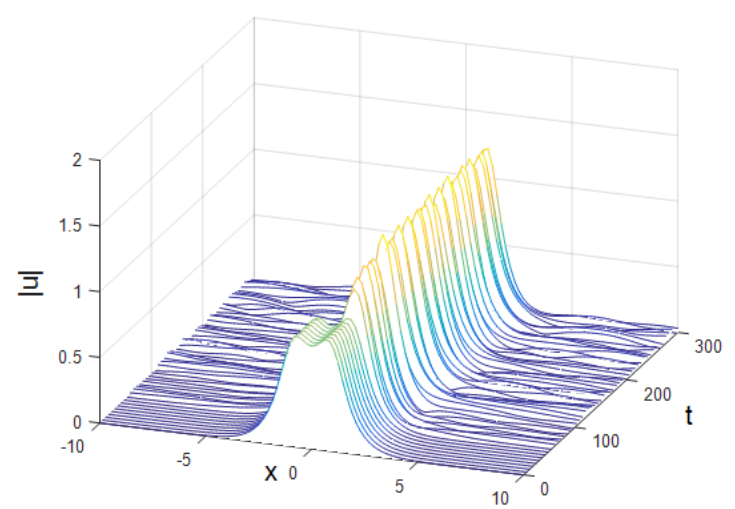

(c)

Fig. 4. The evolution of a stable symmetric state for $N=0.5, a=0.5$ (a), of a stable asymmetric state for $N=2, a=0.5(\mathrm{~b})$, of an unstable symmetric state for $N=2, a=0.5$ (c). The evolution time is $t=300$.

In order to determine the stability of these states, we determine the eigenvalue spectrum of perturbed rate $\lambda$. According to this, if this spectrum contains eigenvalues with positive real parts, the solitary wave is linearly unstable. In the three cases (figures $5,6,7$ ), we find that only in the case of Fig. 7 there exist eigenvalues for the positive real component. We conclude that in the cases of Fig. 5 and Fig. 6 the soliton states are stable, whereas the case of Fig. 7 is the unstable soliton state. The obtained results for the stability of soliton in these cases are the same as the results obtained by two above-mentioned methods. In practice, we concluded that the fastest method is the Method for Linear Stability Eigenvalues. 

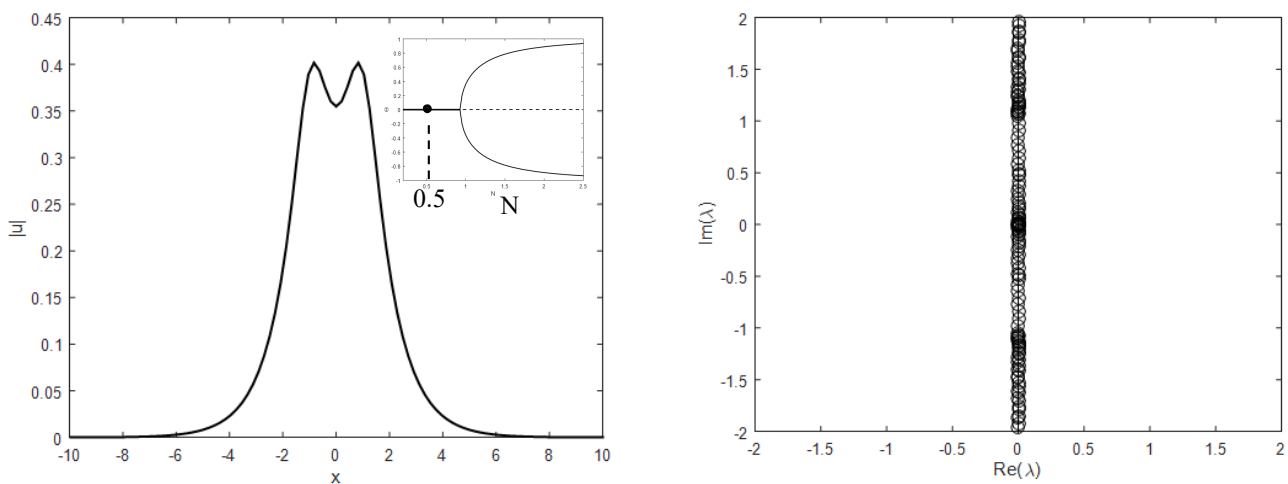

Fig. 5. Example of a stable symmetric state, found with a fixed norm, $N=0.5, a=0.5$. In subplot, the left and right panels show profiles of the stationary states and spectral planes of the instability eigenvalues, respectively.
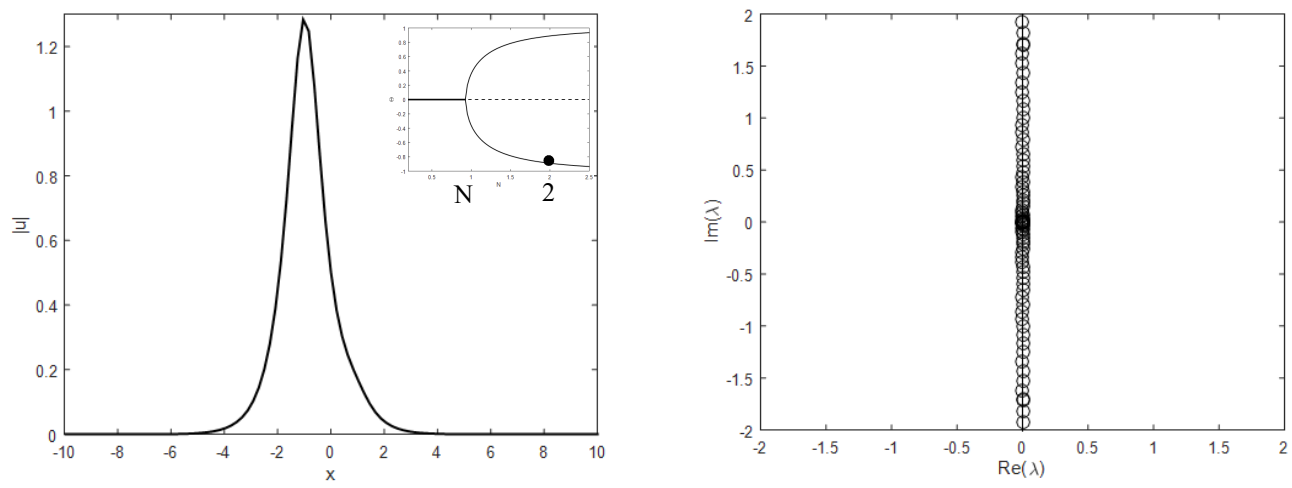

Fig. 6. Example of a stable asymmetric state, found with a fixed norm, $N=2, a=0.5$. In subplot, the left and right panels show profiles of the stationary states and spectral planes of the instability eigenvalues.
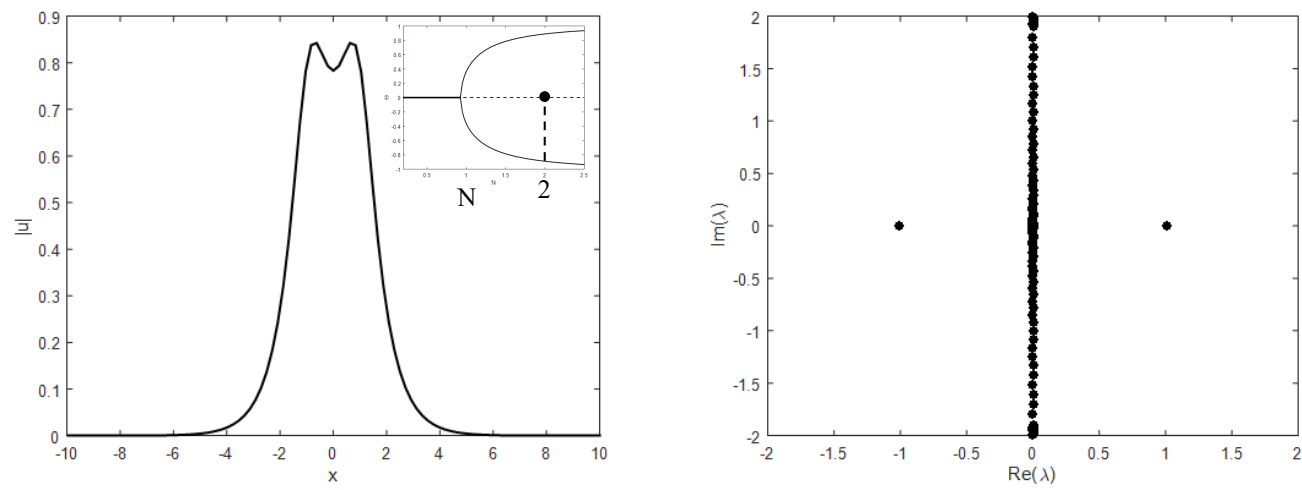

Fig. 7. Example of an unstable symmetric state, found with a fixed norm, $N=2, a=0.5$. In subplot, the left and right panels show profiles of the stationary states and spectral planes of the instability eigenvalues. 
We performed calculations for ten different values of the width $(a)$, we obtained the bifurcate norm as a function of the gaussian width $a$ and bifurcate propagation constant (in optics) or chemical potential (in BEC) as a function of the gaussian width $a$. From the illustration, we determined the regions (1) where stable asymmetry and unstable symmetry states coexist, and the regions (2) where only stable-symmetry states exist.

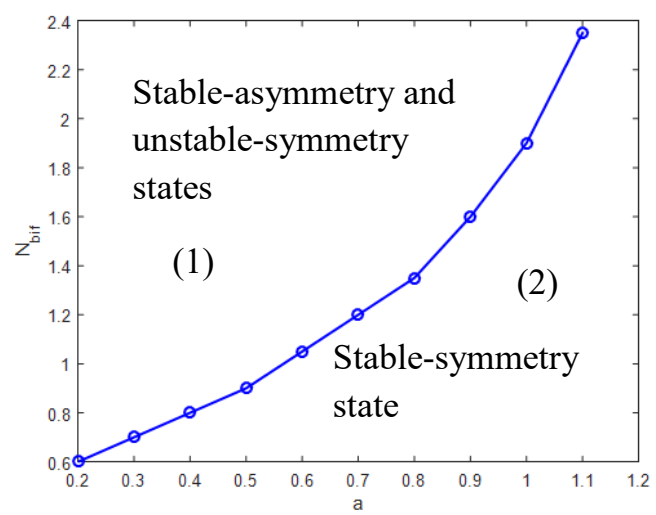

(a)

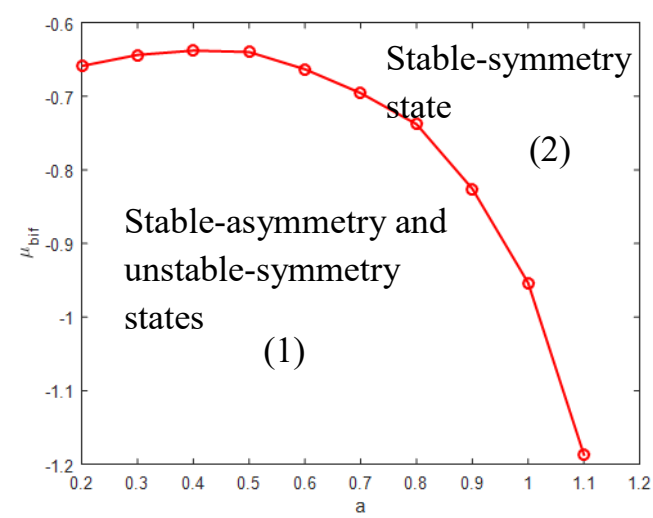

(b)

Fig. 8. (a) shows the coordinates of the bifurcation point, $N_{b i f}$, as functions of $a$, (b) shows the coordinates of the bifurcation point, $\mu_{b i f}$, as functions of $a$.

\section{CONCLUSIONS}

In this work, we have introduced a physical model that gives rise to the SSB of 1D solitons in a dual-core system. This model is extended from a 1D model with a rectangular-shape potential given in Ref. [30] Our model is based on the 1D nonlinear Schrödinger equation with the self-focusing cubic nonlinearity and a 1D double-channel potential. The model applies to the description of spatial optical solitons in a bulk medium with two waveguiding slabs embedded into it, or spatiotemporal solitons in a planar waveguide into which two guiding channels were inserted. The same model may also be interpreted as the Gross-Pitaevskii equation for Bose-Einstein condensate trapped around two attractive parallel light sheets. By using the numerical method we have determined the supercritical symmetry breaking bifurcation in the model We considered also the stability of symmetry and asymmetry states. We obtained the stability regions for these states In particular, we want to emphasize that determining the stability of states in three different ways gives the same result, but the Method for Linear Stability Eigenvalues is the fastest in our case.

Our numerical work in a near future will be extended for considering Coupled Ring Resonators with Gain and Loss [31], where our code used here will be extended to the system of two coupled NLSE.

\section{ACKNOWLEDGEMENT}

One of us (NDC) would like to thank Dr. Nguyen Viet Hung for his useful hints in constructing recent numerical code. 


\section{REFERENCES}

[1] E. B. Davies, Commun. Math. Phys. (England) 64 (1979) 191

[2] B.A. Malomed, I. M. Skinner, P. L. Chu, and G. D. Peng, Physical Review E 53 (1996) 4084

[3] T. Mayteevarunyoo, B. A. Malomed, and G. Dong, Phys. Rev. A 78 (2008) 053601.

[4] M. Trippenbach, E. Infeld, J. Gocaĺek, M. Matuszewski, M. Oberthaler, and B. A. Malomed, Physical review A78 (2008) 013603.

[5] Nguyen Viet Hung, Pawe 1 Ziń, Marek Trippenbach, and Boris A. Malomed, Phys. Rev. E 82 (2010) 046602.

[6] Nguyen Viet Hung, Marek Trippenbach, and Boris A. Malomed, Phys. Rev. A 84 (2011) 053618.

[7] Lazar Gubeskys and Boris A. Malomed, Journal of the Optical Society of America B 30 (2013) 1843.

[8] Nguyen Viet Hung, Marek Trippenbach, Eryk Infeld, and Boris A. Malomed, Physical review A 90 (2014) 023841.

[9] Boris A. Malomed, Spontaneous Symmetry Breaking in Nonlinear Systems: an Overview and a Simple Model, Springer Proceedings in Physics. Vol. 173 (2016).

[10] Elad Shamriz, Nir Dror, and Boris A. Malomed, Phys. Rev. E 94 (2016) 022211.

[11] Krzysztof B. Zegadlo, Nir Dror, Marek Trippenbach, Miroslaw A. Karpierz and Boris A. Malomed, Physical Review A 93 (2016) 023644..

[12] Zhaopin Chen, Yongyao Li, Boris A. Malomed, and Luca Salasnich, Phys. Rev. A 96 (2017) 033621.

[13] Vitaly Lutsky and Boris A. Malomed, Optics Express 25 (2017) 12967.

[14] Krzysztof B. Zegadlo, Nguyen Viet Hung, Aleksandr Ramaniuk, Marek Trippenbach and Boris A. Malomed,Symmetry 10 (2018 156

[15] K. Hayata and M. Koshiba, J. Opt. Soc. Am. B 9 (1992) 1362.

[16] C. Cambournac, T. Sylvestre, H. Maillotte, B. Vanderlinden, P. Kockaert, Ph. Emplit, and M. Haelterman, Phys. Rev. Lett. 89 (2002) 083901.

[17] Y. J. Tsofe and B. A. Malomed, Phys. Rev. E 75 (2007) 056603.

[18] W. C. K. Mak, B. A. Malomed, and P. L. Chu, Phys. Rev. E 55 (1997) 6134

[19] L. Albuch and B. A. Malomed, Math. Comput. Simul. 74 (2007) 312.

[20] F. Dalfovo, S. Giorgini, L. P. Pitaevskii and S. Stringari, Rev. Mod. Phys. 7 (1999) 463

[21] G. J. Milburn, J. Corney, E. M. Wright, and D. F. Walls, Phys. Rev. A 55 (1997) 4318.

[22] A. Smerzi, S. Fantoni, S. Giovanazzi, and S. R. Shenoy, Phys. Rev. Lett. 79 (1997) 4950

[23] M. Matuszewski, B. A. Malomed, and M.Trippenbach,Phys. Rev. A 75 (2007) 063621.

[24] V. M. Pérez-García, H. Michinel, and H. Herrero, Phys. Rev. A 57 (1998) 3837

[25] C. Paré and M. Florjańczyk, Phys. Rev. A 41 (1990) 6287

[26] A. I. Maimistov, Sov. J. Quantum Electron. 21 (1991) 687.

[27] P. L. Chu, B. A. Malomed, and G. D. Peng, J. Opt. Soc. Am. B 10 (1993) 1379.

[28] N. Akhmediev and A. Ankiewicz, Phys. Rev. Lett. 70 (1993) 2395.

[29] A. W. Snyder, D. J. Mitchell, L. Poladian, D. R. Rowland, and Y. Chen, J. Opt. Soc. Am. B 8 (1991) 2102.

[30] JianKe Yang, Nonlinear Waves in Integrable and Nonintegrable Systems, Monographs on Mathematical Modeling and Computation, (2010).

[31] Nguyen Duy Cuong et al, in preparation. 